\title{
$N 89-20339$
}

TDA Progress Report 42-95

July-September 1988

\section{Conceptual Design of a 1-MW CW X-Band Transmitter for Planetary Radar}

\author{
A. M. Bhanji, D. J. Hoppe, B. L. Conroy, and A. J. Freiley \\ Radio Frequency and Microwave Subsystems Section
}

\begin{abstract}
A proposed conceptual design to increase the output power of an existing $X$-band radar transmitter used for planetary radar exploration from $365 \mathrm{~kW}$ to $1 \mathrm{MW} \mathrm{CW}$ is presented. The paper covers the basic transmitter system requirements as dictated by the specifications for the radar. The characteristics and expected performance of the highpower klystron are considered, and the transmitter power amplifier system is described. Also included in the discussion is the design of all of the associated high-power microwave components, the feed system, and the phase-stable exciter. The expected performance of the beam supply, heat exchanger, and monitor and control devices is also presented. Finally, an assessment of the state-of-the-art technology needed to meet system requirements is given and possible areas of difficulty are summarized.
\end{abstract}

\section{Introduction}

Radar has been used as a remote tool for exploration of our solar system since 1946, when detection of echoes from the Moon was reported. Since then, ground-based radar studies have been made of the planets Mercury, Venus, and Mars, the Galilean satellites, the rings of Saturn, and nearly a dozen asteroids. The sensitivity of the radar instruments used in these experiments has increased by a factor of approximately $10^{12}$ since the first lunar detection. Such great gains in sensitivity have been achieved by extraordinary improvements in antenna size, low-noise receiving systems, high-speed digital signal processing, and higher-power transmitters at higher frequencies.
One area that needs further development in order to increase the remote sensing capability of radar is the increase of power in the transmitter. Therefore, at JPL, design and development has been initiated to extend the present X-band transmitter capability from $365 \mathrm{~kW} \mathrm{CW}$ to $1 \mathrm{MW} \mathrm{CW}$.

This experimental transmitter will be installed on the 70meter dual-reflector antenna at Goldstone, California, which is equipped with a rotatable, asymmetric subreflector that permits the use of multiple feed systems at the antenna focus. The subreflector can be precision indexed to a fixed number of positions that will allow each feed to properly illuminate the main reflector. Assuming an aperture efficiency of about 
$75 \%$ and corresponding antenna gain of $74.5 \mathrm{~dB}$ at X-band, with the proposed 1-MW $\mathrm{CW}$ transmitter, the $\mathrm{X}$-band radar system will have an effective radiated power of about $28 \mathrm{TW}$ $\left(2.8 \times 10^{13} \mathrm{~W}\right)$.

Using an assessment of the state-of-the-art technology, this article describes the $\mathrm{X}$-band transmitter, including the transmission system and a feed system.

\section{Transmitter System Requirements}

The transmitters used for radar astronomy systems differ from conventional radar systems in that they require high average power, rather than high peak power, over the bandwidth required to handle the transmitted signal [1]. It is also important that these transmitters be coherent in order to determine the phase relationships of the returned signals, and they must have high phase stability if coherent measurements are to be made over long periods of time. The transmitter must also be capable of modulation by a variety of pulse programs, while maintaining the phase and amplitude fidelity and pulse-to-pulse stability required for pulse-compression systems incorporated in the radar.

The above requirements illustrate that high power alone will not provide the desired $\mathrm{CW}$ radar transmitter capabilities. If this were the case, it might be more easily obtained with an oscillator rather than an amplifier. Besides the advantage of having dynamic control of amplitude and phase, the appeal of using an amplifier is that it eliminates the need for phaselocking an oscillator to a control signal.

Based on the above requirements, the $\mathrm{X}$-band radar transmitter specifications are given in Table 1.

\section{The Transmitter System}

As shown in Fig. 1, the transmitter will include a power supply that converts $2400-\mathrm{V}, 3 \cdot$ phase, $60-\mathrm{Hz}$ line voltage to direct current at up to $50 \mathrm{kV}$ with a power limitation of $2.25 \mathrm{MW}$ for the four klystron amplifier beams. The frequency synthesizer-based exciter and the buffer amplifier will provide an input signal to these klystrons, and each of the four klystrons will provide approximately a $53-\mathrm{dB}$ power gain. The automated transmitter control will perform monitoring and control of all functions. Protective devices (interlocks) will prevent damage to equipment by removing voltage and in some cases drive power in the event of a malfunction. The liquid-to-air 2.5-MW heat exchanger will be used to cool the amplifier, the power supply, various auxiliaries to the transmitter, microwave components of the transmitter, and microwave components of the transmission line. The following para- graphs describe each of the above components in greater detail.

\section{A. Exciter}

Figure 2 shows the proposed configuration of the exciter portion of the 1-MW radar, which is based on an HP 8662A synthesizer. The synthesizer uses the $10-\mathrm{MHz}$ reference signal from a hydrogen maser to produce a phase-coherent output at $640 \mathrm{MHz}$, and a phase-coherent variable-frequency signal from $10 \mathrm{kHz}$ to $640 \mathrm{MHz}$ with $0.1-\mathrm{Hz}$ resolution, or from 640 to $1280 \mathrm{MHz}$ with $0.2 \cdot \mathrm{Hz}$ resolution. The $640-\mathrm{MHz}$ signal can be picked off and mixed with the variable-frequency output. For the transmitter, the variable-frequency output is set to 830 $\mathrm{MHz}$ and mixed with 12 times the $640 \mathrm{MHz}$ to produce 8510 $\mathrm{MHz}$. In the receive configuration, the variable frequency is set to $505 \mathrm{MHz}$ and mixed with 12 times the $640 \mathrm{MHz}$ to produce a signal at $8185 \mathrm{MHz}$, which could be used as the first local oscillator in the receiver. A similar system, using 3 times $640 \mathrm{MHz}$, is used for the S-band exciter and could also be used as a first local oscillator for an S-band receiver.

Reduction of phase noise is a major concern in the exciter design. By mixing the output of an extremely low-noise, highfrequency synthesizer with low multiples of a clean, fixedfrequency oscillator, total phase noise should be reduced by more than $20 \mathrm{~dB}$ from that produced by the more standard method of using a high multiple of a low-frequency synthesizer.

Provisions are made for biphase noise modulation, frequency hopping, and continuous frequency sweep for Doppler cancellation. The frequency hopping is accomplished by sending frequency step commands to the synthesizer on an IEEE488 interface. Doppler cancellation is done through a combination of a coarse frequency, set through the IEEE-488 interface, and an analog voltage at the search oscillator input. Phase noise modulation is done directly with a biphase modulator at the output frequency.

A power divider is included in the exciter, providing separate outputs for the phase reference system and the klystron drive system in the buffer amplifier.

\section{B. Buffer Amplifier}

The buffer amplifier is the second major block of Fig. 2 . For the first choice of transmission line arrangements (see Section D), phase-tracking loops and electronic polarization control are provided in the exciter. The phase-tracking loop uses a voltage-tracking controlled phase shifter in the drive path of each klystron to compensate for phase changes in klystrons or microwave components. A sample of the output from each 
klystron is taken as close to the feedhorn as possible and compared with the reference signal. Symmetry in the waveguide paths from the final splitter is still required to prevent differential phase shift between the two horn inputs driven by the same klystron.

Because the feed system uses two klystrons to drive the in line inputs to the horn and the other two klystrons to drive the orthomode inputs, polarization control is achieved by the phase shifter after the first splitter in the buffer amplifier. Phase shifts of \pm 90 degrees yield right-hand or left-hand circular polarization, and phase shifts of 0 or 180 degrees yield orthogonal linear polarizations. Other phase shift values produce various elliptical polarizations. A corresponding phase shift must be introduced in the phase correction loop, but in the case of switching from right-hand circular to left-hand circular polarization, this can be done with an inverting amplifier after the phase detector. For the waveguide-based system, no special electronic control is required in the exciter. In both cases, a solid-state amplifier for each klystron provides the approximately 2 -watt drive power required.

\section{Power Amplifier}

The power amplifier section of the transmitter contains four $250-\mathrm{kW} \mathrm{CW}$ klystrons. The requirements of high power, high gain, good efficiency, ease of modulation, and an output spectrum free from spurious signals and noise make a klystron linear-beam tube the natural choice for radars, as long as its narrow bandwidth, high operating voltage, and large size can be tolerated.

Early in 1986, Varian Associates was contracted by JPL to do a paper design for eventual development and production of extremely high-power $\mathrm{X}$-band klystrons. The characteristics of this tube, designated VKX-7864A, are given in Table 2.

As part of the design for this klystron, Varian is expected to provide phase modulation sensitivity due to various pushing factors such as beam voltage, drive power, body coolant, focus current, and heater voltage. These phase-pushing factors are important because they produce unwanted discrete lines on the phase noise spectrum.

Based on overall transmitter system specifications, these modulation sensitivities, together with modulation sensitivity of other pushing factors, establish the requirements for power supply stability, body coolant temperature stability and stabilities of other pushing (control) factors.

Each klystron is provided with an arc detector at the window and a reverse power coupler for protection. In the event of a fault, these monitors will remove the RF drive before permanent damage can occur.

One of the critical elements of the klystron is the guiding magnet. This device is a solenoid which surrounds the interaction volume and keeps the electron beam focused in the tube length before the collector. A control of better than $1 \%$ must be exercised to maintain high efficiency and low body current. This solenoid will weigh about 300 pounds and will require a $300-\mathrm{V}, 20-\mathrm{A} \mathrm{dc}$ power supply to provide the proper magnetic field.

The separate coolant manifold for each klystron will monitor and control flows, temperature, and pressure. This data will be routed to the data collection system, which is described in Section $\mathrm{H}$.

The power amplifier, including the transmission line (described in the next section) will all be housed in the cone. The mechanical layout is shown in Fig. 3.

\section{Transmission Line}

Two alternatives for the transmission line system are under consideration. The first system is shown in Fig. 4. For this system, the output from each klystron passes through a waveguide switch and a directional coupler before being split into two $125-\mathrm{kW}$ signals. Four of these signals (two pairs) feed the in-line ports of four orthomode junctions, while the other pair feed the ortho ports. Thus, by adjusting the relative phase between the two pairs of klystrons, one of two orthogonal linear polarizations, RHCP or LHCP, may be obtained (see buffer amplifier, Section C). The outputs of the four orthomode junctions then feed the four inputs to the multimode feed. horn. In this system, phase detectors and electrically controlled phase shifters will be used to adjust the outputs of each of the klystrons and to provide polarization control. The reliance on electronics reduces the complexity of the waveguide layout in comparison to the waveguide-based system described below.

Figure 5 shows the waveguide-based alternative to the previous system. This system is similar to that used at the Haystack Hill Observatory in Westford, Mass. [2]. In this system, a series of splitters and combiners ultimately forms four identical signals. Each of the signals is composed of $25 \%$ of the power from each klystron, and all four are equal in amplitude and phase, independent of the four klystron outputs. Phase shifters are used in each of the drive lines to the klystrons in order to minimize the power in the waster loads. This adjustment is made once, and if klystron parameters or frequencies change during a track, only the waster-load power will change. 
Polarization is changed mechanically through switches immediately before the orthomode junctions. Although this ap. proach is complicated mechanically, it has the advantage that beam position and polarization purity are virtually guaranteed without the use of any electronics.

The final decision on which of the two possible systems, or a combination thereof, will be used depends on how closely the four klystron tubes can be matched in terms of phase, gain, and group delay versus frequency. Measurements on the two existing tubes as well as discussions with the tube manufacturer (Varian Assoc.) are underway to help answer these questions. The effects of aging must also be considered to guarantee that the system will run reliably over the expected lifetime of the tubes with minimal adjustment.

In both systems, WR 125 is used as the high-power waveguide to avoid operation close to the higher-order modes in WR137, which begin propagating at $8600 \mathrm{MHz}$ [3] .

The electrically operated waveguide switches allow selection of the radar antenna or water loads for termination of transmitter output power. The water loads will also be used for calibrating the output power calorimetrically.

On receive, the klystrons are turned off and the switches are rotated, connecting the receive waveguide to the feed. horn. Through a series of combiners, RHCP and LHCP signals are formed. These signals then enter the dual-channel maser, and finally the heterodyne receiver.

In addition, the existing low-noise system, which uses a separate corrugated horn for receiving, will be retained. The disadvantage of this receiving arrangement is that the antenna subreflector must be rotated between transmit and receive cycles.

\section{E. Antenna Feed System}

The final component required in the transmission line for the radar system is the feedhorn, which will launch the transmitter power toward the subreflector of the antenna. The horn should illuminate the subreflector efficiently and increase the overall noise temperature of the system as slightly as possible. The horn will be designed to meet or exceed the RF performance of the feedhorns presently in use, with the added feature of 1-MW capability.

Several possible feed types were considered for the 1-MW system. Experience indicates that conventional corrugated or dual-mode horns are not capable of handling the large $\mathrm{CW}$ power without breaking down at the small-diameter input section. It was also found that a rather large number of small horns arranged in a closely packed array would be required to illuminate the subreflector efficiently. Due to the complexity of this type of system, as well as the losses associated with the power-splitting components, the array concept was also rejected for the $1-\mathrm{MW}$ system.

The chosen design uses a multiflare rectangular horn [4]. Such a horn is well suited to the 1-MW system, since it possesses an excellent radiation pattern and has been used in other power applications.

The multimode horn is depicted in Fig. 6. Four square waveguides feed a large square chamber where the power is launched into a square multiflare horn. Since the large chamber is oversized for the frequency of interest, the sum of the power in the four waveguides can be supported without breakdown. In the present case, each of the guides must support $250 \mathrm{~kW}$, and the large chamber $1 \mathrm{MW}$. Flare angle changes are used to generate the required higher-order modes for pattern symmetry.

The analysis of the horn is carried out using mode-matching methods. The overall scattering matrix of the horn is obtained and, using these results, the input match, as well as the farfield radiation pattern, can be predicted for arbitrary input levels and phases in the four input guides. Calculated radiation patterns for the horn at $8.51 \mathrm{GHz}$ are shown in Fig. 7. A detailed description of the horn will be provided in a future report.

Estimates for the peak electric fields in the horn indicate that the maximum level (about $6.9 \mathrm{kV} / \mathrm{cm}$ ) occurs in the four feeding waveguides, which are $0.95 \mathrm{in}$. square. This should be compared to the present 400-kW WR125 waveguide system (about $8.5 \mathrm{kV} / \mathrm{cm}$ ) and the theoretical limit for a 0.95 -in.square waveguide, which is about $2.1 \mathrm{MW}$. Resonant ring tests [5] will be used to evaluate the power performance of the orthomode junctions and the horn. Should arcing become a problem, backup approaches include evacuating areas of the horn or pressurizing them with a dielectric gas such as $\mathrm{SF}_{6}$.

\section{F. Beam Power Supply}

A block diagram of the beam supply is shown in Fig. 8 . Power at $12,600 \mathrm{~V}, 3$ phase, and $60 \mathrm{~Hz}$ is supplied to separate substations from the commercial line (Edison), which is underground for the last mile. The $2400 \cdot \mathrm{V}$ substation supplies the main motor generator only. Under critical operation, this $2400-\mathrm{V}, 60-\mathrm{Hz}$ power is supplied separately by the diesel generator. All auxiliaries are supplied from a $480 . \mathrm{V}$ substation. The output of the main motor-generator at $400 \mathrm{~Hz}$ is stepped up in voltage, transformed, rectified, and delivered to the klystron load through a filter, crowbar, and series limiter 
resistor to voltages of $50 \mathrm{kV}$ and $2.25 \mathrm{MW}$. The design of this power supply will maintain output ripple under full load of less than $0.05 \%$, regulation of $0.01 \%$, and settling time of 200 milliseconds.

The use of a frequency converter (such as a motor-generator) might seem unnecessary, but it actually provides worthwhile technical and economic advantages. It isolates the power line from a crowbar of the dc supply and greatly simplifies line protection problems. It also isolates the supply from short. duration line voltage fluctuations and transients due to the large inertia of the rotating components. The change from 60 to $400 \mathrm{~Hz}$ reduces all transformer and filter sizes and costs.

This beam supply is required to provide $50 \mathrm{kV}$ between the klystron collector and cathode at a beam current of $11 \mathrm{amps}$ (per klystron) during the long radar pulses (up to 10 hours).

The ability of the beam supply to remain ripple free and tightly regulated, and to settle in $200 \mathrm{msec}$ will require a unique and state-of-the-art feedback control circuit. The design is in progress and the first approach is being tested. The supply must also be capable of withstanding the stress imposed on it when an arc occurs in any klystron.

\section{G. Cooling System}

The cooling system provides a $2.5-\mathrm{MW}$ cooling capacity for klystrons, focusing magnets, high-power microwave components, water loads, feed, and the transformer rectifier, including the motor-generator clutch. Basically, the cooling system is a closed loop which consists of a heat exchanger, a distribution manifold, and all connecting piping. Coolant is circulated through the cooling system by the heat exchanger pumps. The coolant gains heat as it passes through the RF system (buffer amplifier, power amplifier, and microwave components) and loses heat as it passes through the heat exchanger. A purity loop is connected to the input of the heat exchanger to maintain the purity of the coolant.

As part of this transmitter modification, the pumps will have to be upgraded, including replacing all of the 6-inch water lines, and a complex water-switching mechanism will have to be installed. The water-to-water heat exchanger will have to be changed to a liquid-to-air heat exchanger.

\section{H. Monitor and Control}

Operation of the 1-MW radar will be extensively automated. The control system will be composed of an HP Industrial
Vectra computer, two HP 38526 Data Acquisition and Control units, a frequency counter, and a multichannel power meter. All the instruments will be connected by an IEEE-488 interface bus, with a fiber optic extension between the control room and the cone area of the antenna. The IEEE bus will also connect to the synthesizer in the exciter.

The monitor and control software will be written in Ada, a language especially designed for hardware control applications, and will make use of artificial intelligence principles to maximize the system functionality while maintaining a simple user interface. It will automatically keep long-term data logs to look for trends and predict failures before they can disable the radar. It will correlate data from different sources to distinguish between sensor problems and transmitter problems, and it will be able to calibrate the RF power meters by precision measurement of the flow rates and temperature rises of the coolant in the water loads.

\section{Concerns and Conclusion}

The previous sections make it clear that this radar transmitter will be a very complicated system, and therefore there are several areas of concern. Of primary concern is the possibility of waveguide/feedhorn breakdown. Associated with this problem is the difficulty of testing the components at or above the normal operating power level before all of the klystrons arrive. Resonant ring testing can be used for some components, but the most critical component, the feedhorn, can be tested under full power only when all four klystrons are available.

Several additional areas of concern involve the klystron tubes. Both systems under consideration, particularly the transmission line system (Option 1), demand that the four amplifiers have matched gain and phase characteristics over the band of interest. This tube-to-tube matching could become difficult, particularly given the stretched procurement schedule for these tubes. Also, in order for the radar to operate at full output power, a VSWR of less than 1.05 to 1 must be presented to each tube. This is a difficult requirement. The feedhorn window, which must be capable of handling $1 \mathrm{MW} \mathrm{CW}$, is also a concern. If the present material (kapton) is not suitable, an alternative must be found.

Finally, this radar transmitter would be the most complicated transmitter in the field, with the most densely populated feedcone on the 70-meter antenna, and would require special knowledge and care from the maintenance personnel at the site. 


\section{References}

[1] S. A. Hovanessian, Radar Detection and Tracking Systems, Dedham, Massachusetts: Artech House, Chapter 11, Section 5, pp. 11-22, 1973.

[2] W. North, "Haystack Hill Long Range Imaging Radar Transmitter," Proceedings of the 13th Pulse Power Modulator Symposium, pp. 247-253, 1978.

[3] H. R. Buchanan, X-Band Uplink Microwave Components, JPL Technical Report 32-1526, vol. XII, Jet Propulsion Laboratory, Pasadena, California, pp. 22-24, December 15, 1972.

[4] K. R. Goudey and A. F. Sciambi, Jr., "High Power X-Band Monopulse Tracking Feed for the Lincoln Laboratory Long Range Imaging Radar," IEEE Trans. Microwave Theory Tech., vol. MTT-26, no. 5, pp. 326-332, May 1978.

[5] D. J. Hoppe and R. M. Perez, "X-Band Resonant Ring Operation at $450 \mathrm{~kW}$," TDA Progress Report 42-93, vol. January-March 1988, Jet Propulsion Laboratory, Pasadena, California, pp. 18-26, May 15, 1988. 
Table 1. 1-MW X-band radar transmitter specifications

\begin{tabular}{|c|c|}
\hline Parameter & Specification \\
\hline Frequency & $8.51 \mathrm{GHz}$ \\
\hline Bandwidth & $\begin{array}{l}20 \mathrm{MHz}(-1 \mathrm{~dB}) \\
6 \mathrm{MHz} \text { (normal usable range) }\end{array}$ \\
\hline RF output power & $1 \mathrm{MW} \mathrm{CW}(+90 \mathrm{dBm})$ \\
\hline RF stability & $\begin{array}{l} \pm 0.25 \mathrm{~dB} \text { over one planetary transmit/ } \\
\text { receive cycle }\end{array}$ \\
\hline Incidental AM & $\begin{array}{l}60 \mathrm{~dB} \text { below carrier at all modulating } \\
\text { frequencies above } 1 \mathrm{~Hz}\end{array}$ \\
\hline Phase stability & $10^{-15}(1000 \mathrm{sec})$ \\
\hline Incidental PM (jitter) & $<1^{\circ}$ peak to peak \\
\hline Transmit period & $30 \mathrm{sec} \min$. to $10 \mathrm{hr} \max$. \\
\hline \multicolumn{2}{|l|}{ Modulation: } \\
\hline Phase Modulation & $\begin{array}{l}\text { Biphase, } 40-\mathrm{dB} \text { carrier suppression, } \\
\mathrm{dc} \text { to } 20 \mathrm{MHz}\end{array}$ \\
\hline $\begin{array}{l}\text { Frequency } \\
\text { Hopping }\end{array}$ & $\pm 2 \mathrm{MHz}$ every few seconds \\
\hline $\begin{array}{l}\text { Frequency } \\
\text { Ramping }\end{array}$ & $\pm 2 \mathrm{MHz}$ in $200 \mathrm{msec}$ \\
\hline Group Delay Dispersion & $10 \mathrm{nsec}$ over $6-\mathrm{MHz}$ bandwidth \\
\hline Polarization & $\begin{array}{l}\text { RHCP or LHCP (one polarization at a } \\
\text { time; cross polarization }<-25 \mathrm{~dB} \text { ) }\end{array}$ \\
\hline
\end{tabular}

Table 2. Characteristics of VKX-7864A X-band klystron

\begin{tabular}{ll}
\hline Parameter & \multicolumn{1}{c}{ Specification } \\
\hline Frequency & $8510 \mathrm{MHz}$ \\
Bandwidth & $20 \mathrm{MHz}$ (1-dB points) \\
Output power & $250 \mathrm{~kW} \mathrm{min.}$ \\
Beam voltage & $50 \mathrm{kV}$ \\
Beam current & $11 \mathrm{~A}$ \\
Efficiency & $50 \%$ \\
Gain (sat) & $53 \mathrm{~dB}$ \\
Filament voltage & $15 \mathrm{~V}$ \\
Filament current & $20 \mathrm{~A}$ \\
Magnet voltage & $300 \mathrm{~V}$ \\
Magnet current & $20 \mathrm{~A}$ \\
Klystron weight & $300 \mathrm{lb}$ \\
Klystron height & $5 \mathrm{ft}$ \\
\hline
\end{tabular}




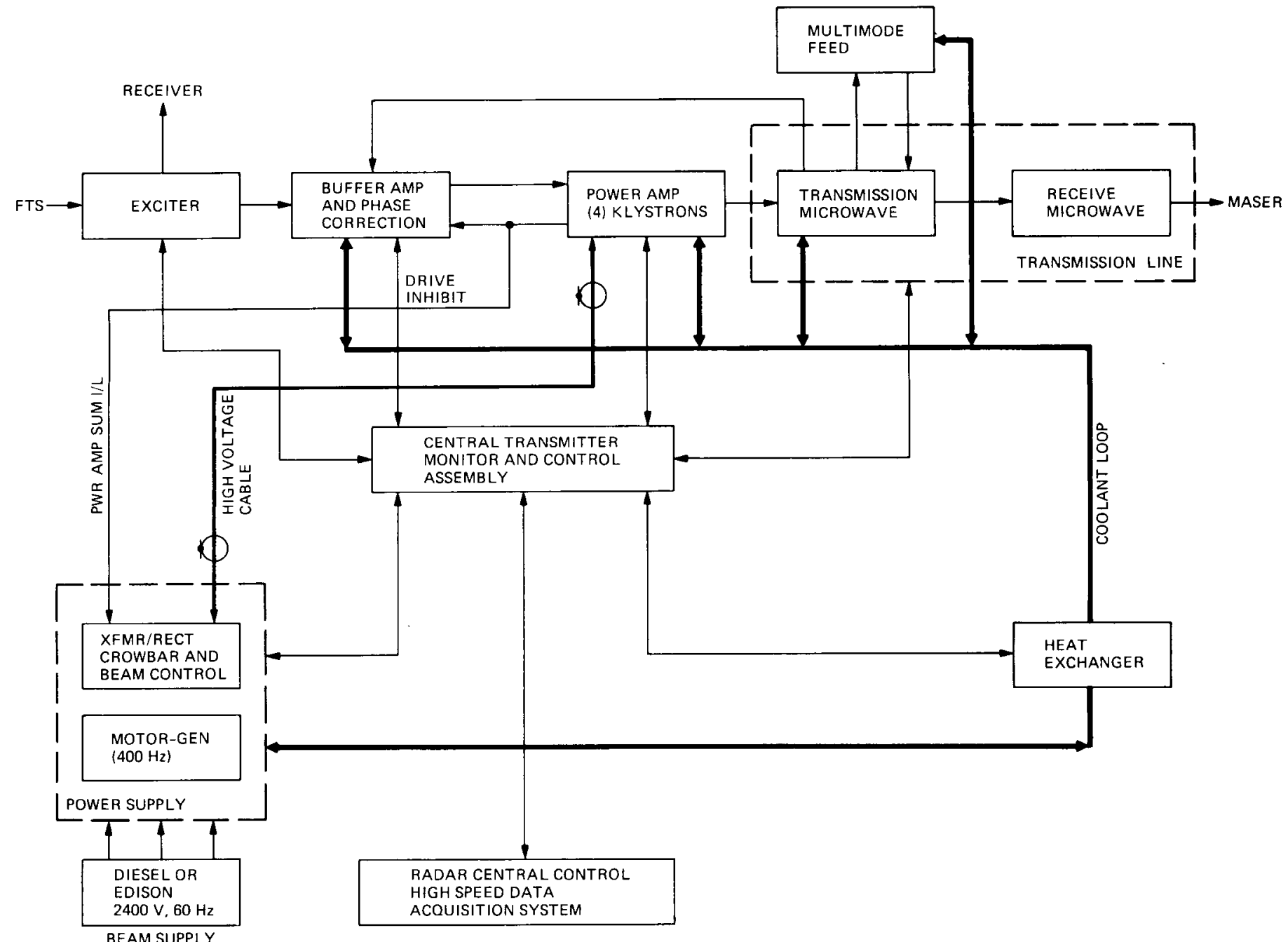

Fig. 1. 1-MW X-band radar transmitter block diagram. 


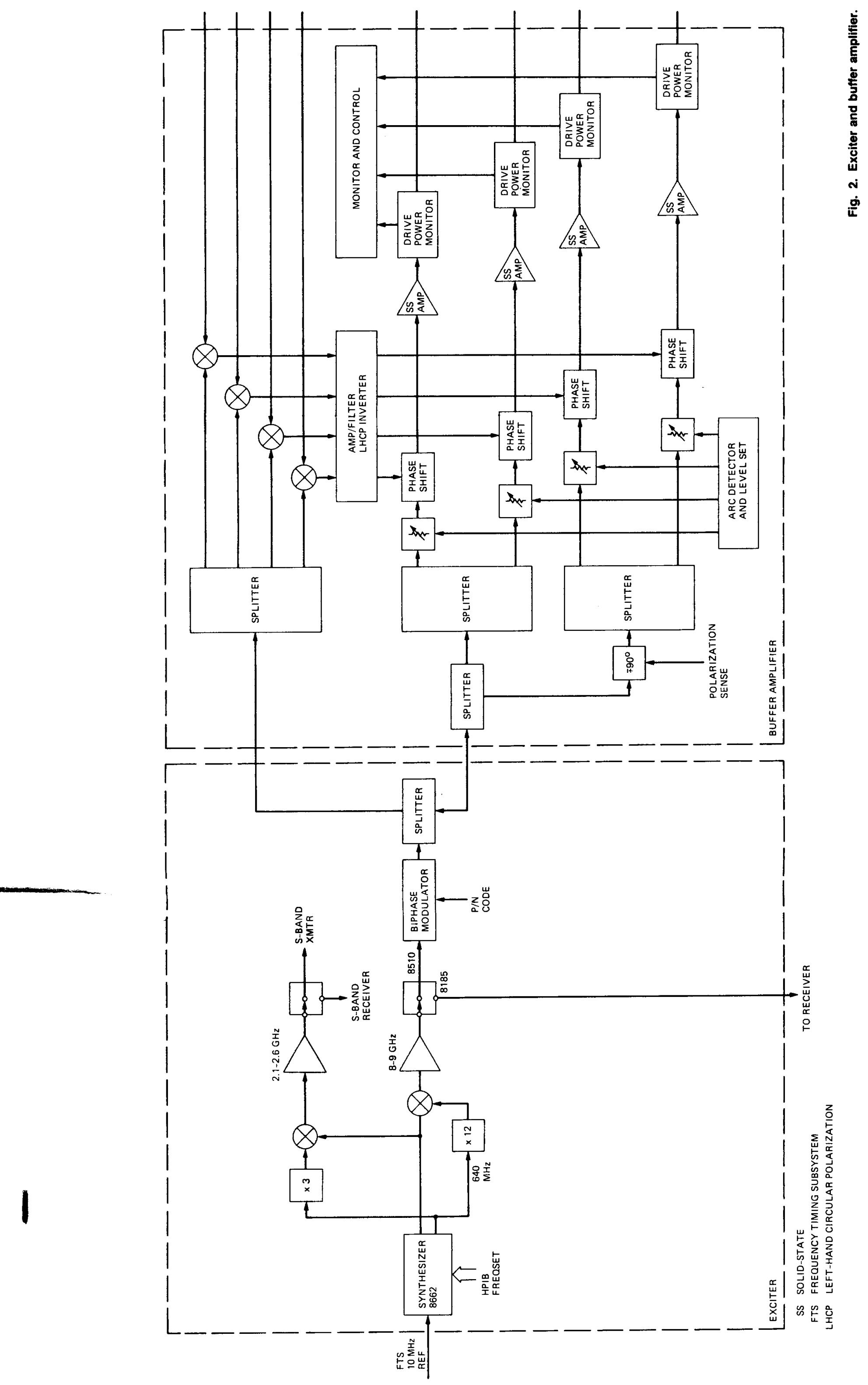

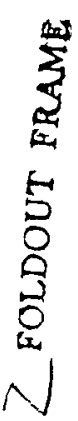




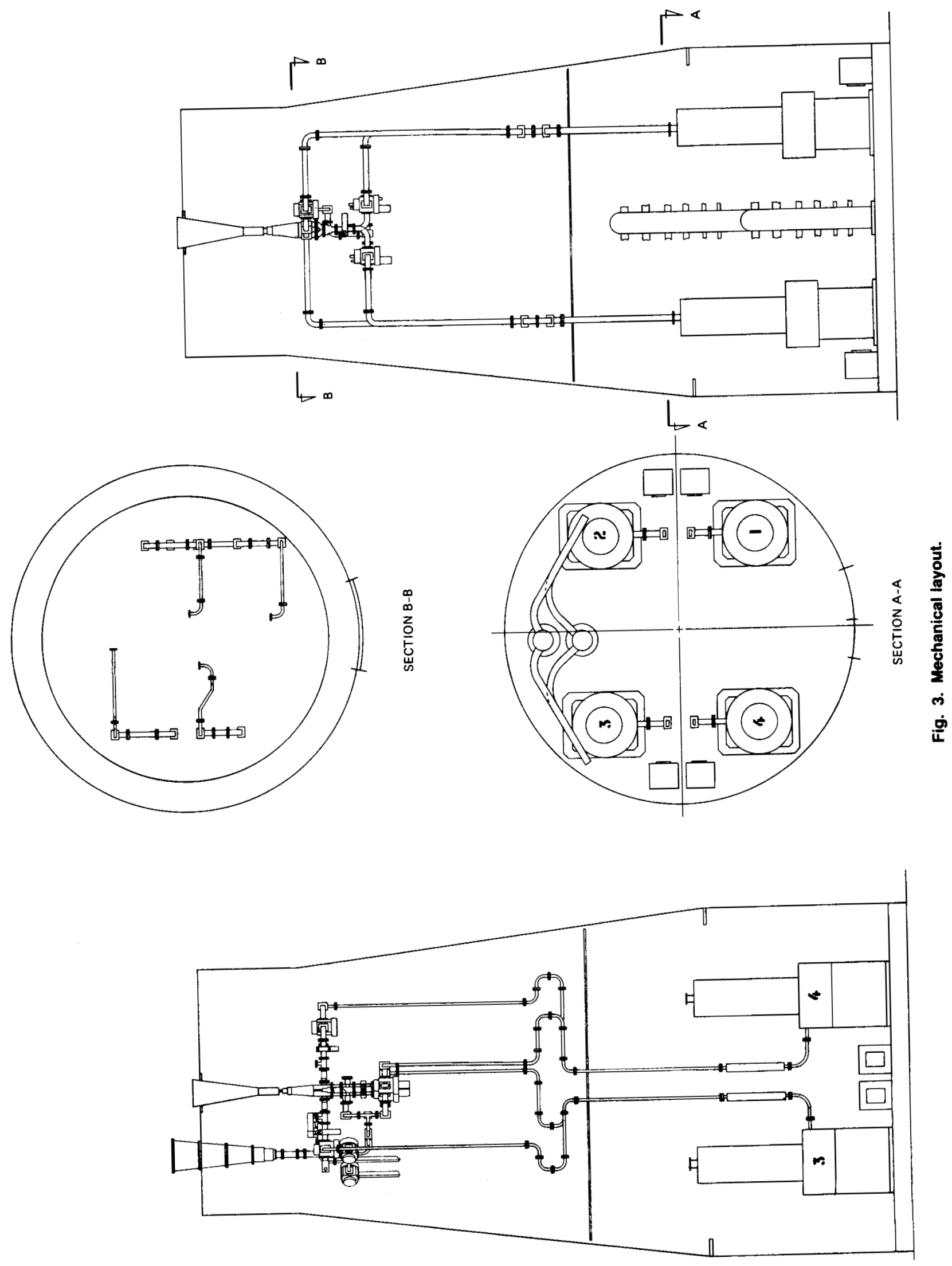




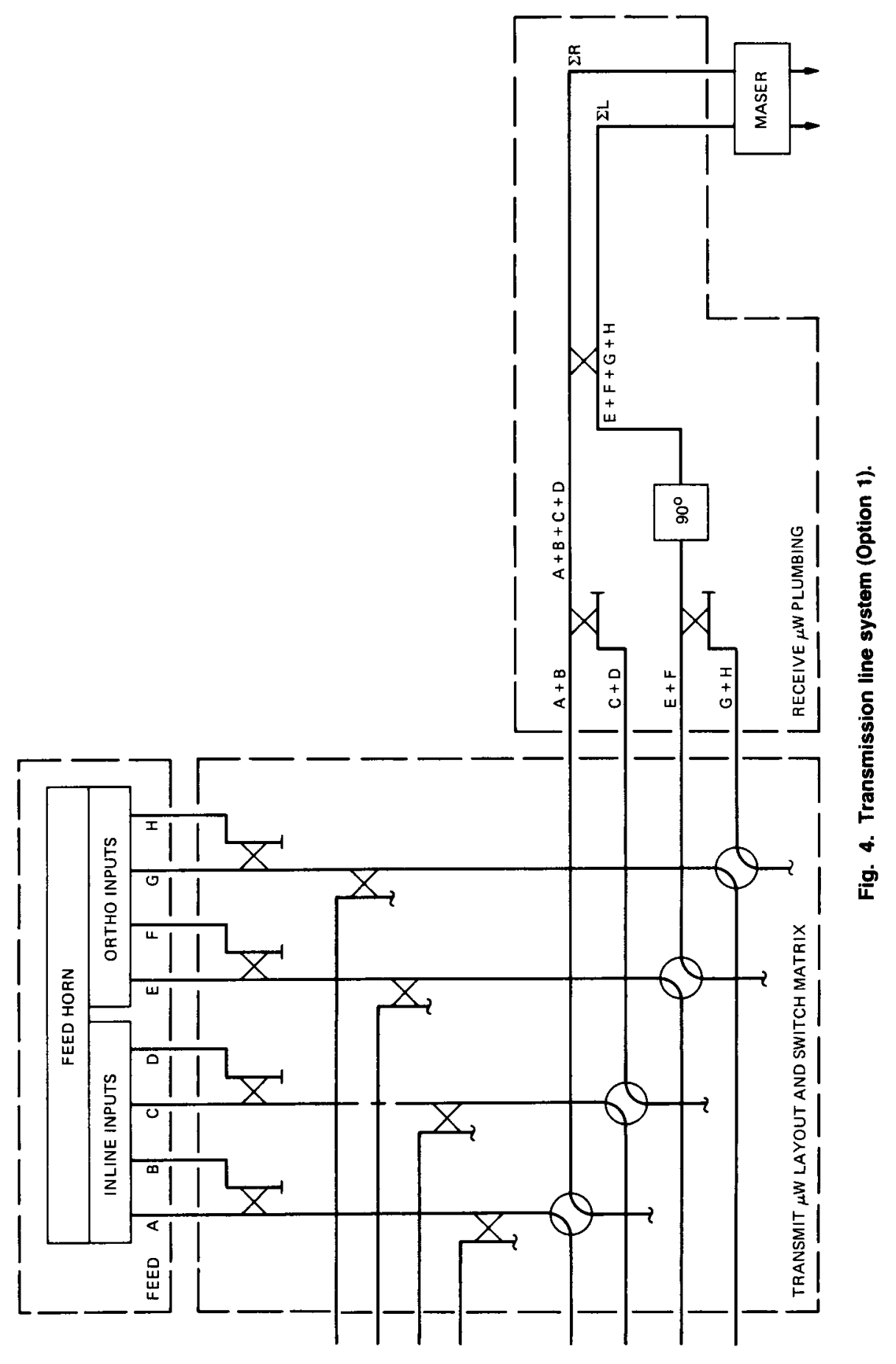




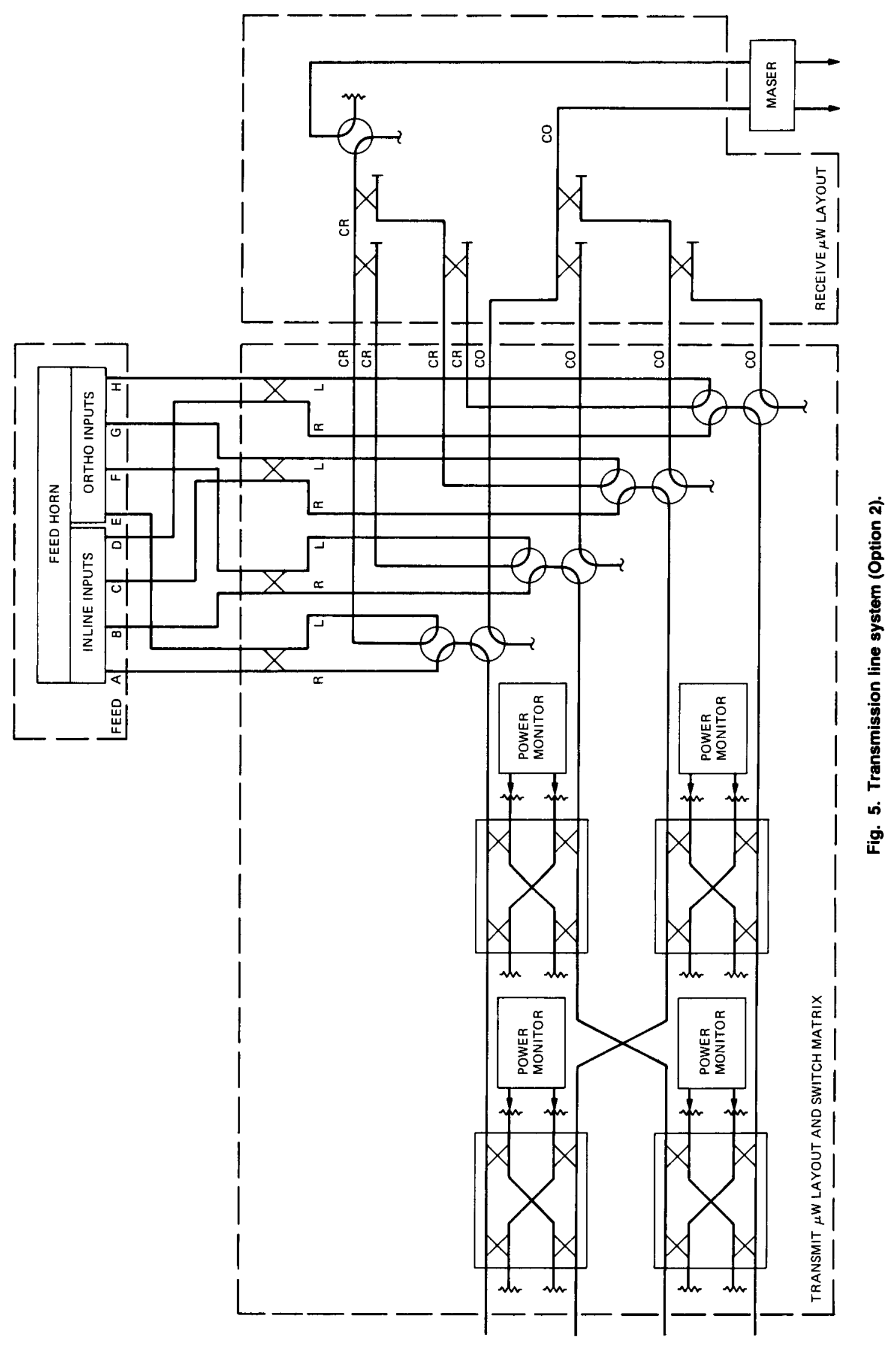




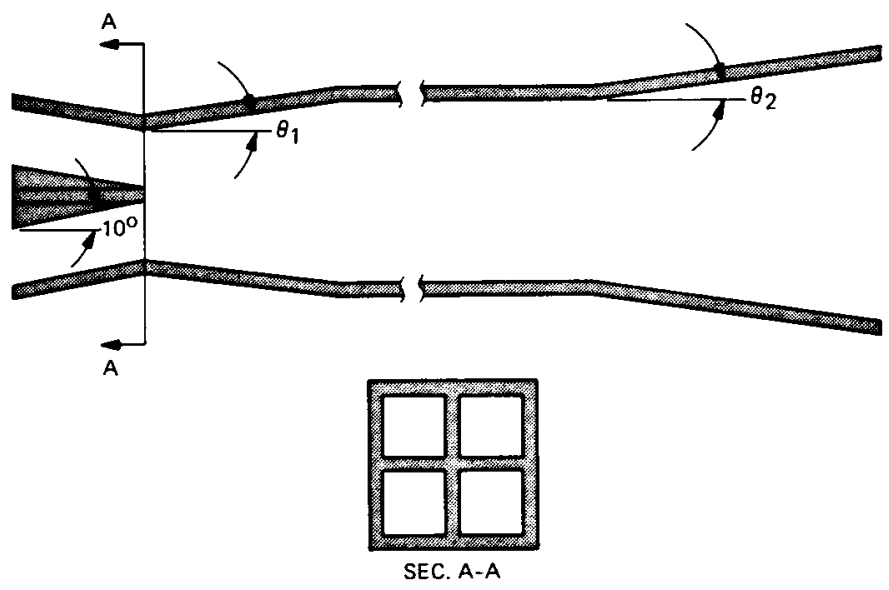

Fig. 6. 1-MW multiflare horn.

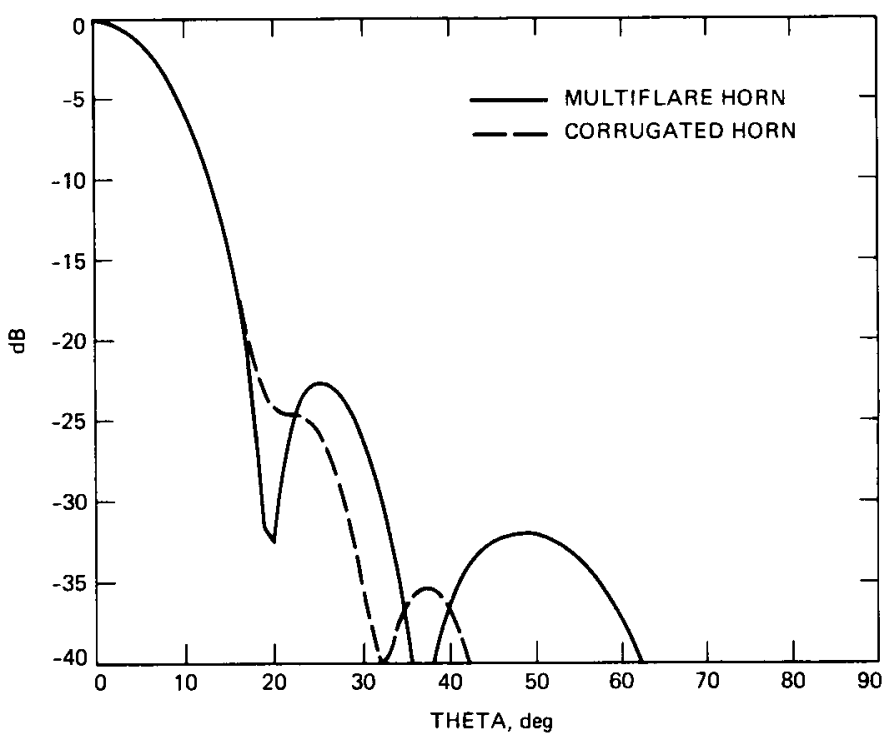

Fig. 7. 1-MW multiflare horn patterns at $8.51 \mathrm{GHz}$. 


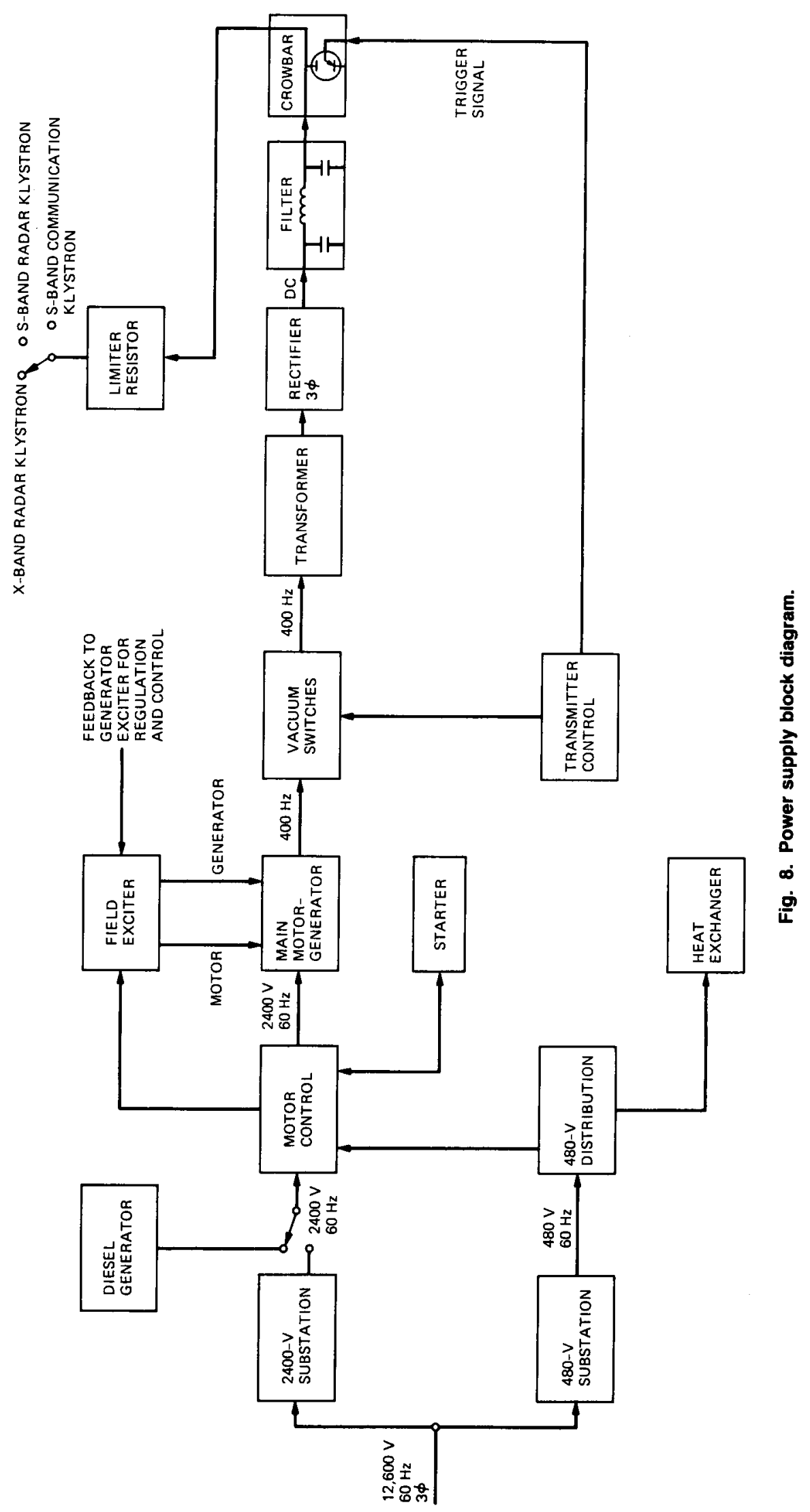

